\title{
GENERALIZED FOURIER-WIENER FUNCTION SPACE TRANSFORMS
}

\author{
Seung Jun Chang And Hyun Soo Chung
}

\begin{abstract}
In this paper, we define generalized Fourier-Hermite functionals on a function space $C_{a, b}[0, T]$ to obtain a complete orthonormal set in $L_{2}\left(C_{a, b}[0, T]\right)$ where $C_{a, b}[0, T]$ is a very general function space. We then proceed to give a necessary and sufficient condition that a functional $F$ in $L_{2}\left(C_{a, b}[0, T]\right)$ has a generalized Fourier-Wiener function space transform $\mathcal{F}_{\sqrt{2}, i}(F)$ also belonging to $L_{2}\left(C_{a, b}[0, T]\right)$.
\end{abstract}

\section{Introduction}

Let $C_{0}[0, T]$ denote one-parameter Wiener space, that is the space of realvalued continuous function $x$ on $[0, T]$ with $x(0)=0$. The concept of the Fourier-Wiener transforms was introduced by Cameron and Martin in [2]. In [3], the authors defined a modified Fourier-Wiener transform and gave various relationships for the modified Fourier-Wiener transform of functionals in $L_{2}\left(C_{0}[0, T]\right)$. For these works, in [4], using the Wiener measure on $C_{0}[0, T]$ and completeness properties of the Hermite polynomials on $\mathbb{R}$, they introduced a complete orthonormal set in $L_{2}\left(C_{0}[0, T]\right)$ and gave a Fourier development for functionals in $L_{2}\left(C_{0}[0, T]\right)$ which converges in the $L_{2}\left(C_{0}[0, T]\right)$.

The function space $C_{a, b}[0, T]$ induced by generalized Brownian motion was introduced by J. Yea in [16] and was used extensively by Chang and Chung [7]. In this paper, we extend the results of [1-4] to a very general function space $C_{a, b}[0, T]$ rather than the Wiener space $C_{0}[0, T]$. The Wiener process used in $[1-6,10]$ is stationary in time and is free of drift while the stochastic process used in this paper as well as in [7-9], in nonstationary in time, is subject to a drift $a(t)$, and can be used to explain the position of the Ornstein-Uhlenbeck process in an external force field [14]. However, when $a(t) \equiv 0$ and $b(t)=t$ on $[0, T]$, the general function space $C_{a, b}[0, T]$ reduces to the Wiener space $C_{0}[0, T]$.

Received July 31, 2007.

2000 Mathematics Subject Classification. 28C20, 60 J65.

Key words and phrases. generalized Brownian motion process, generalized Hermite function, generalized Fourier-Hermite coefficient, generalized Fourier-Wiener function space transform.

The present research was conducted by the research fund of Dankook University in 2006. 


\section{Definitions and preliminaries}

Let $D=[0, T]$ and let $(\boldsymbol{\Omega}, \mathcal{B}, P)$ be a probability measure space. A realvalued stochastic process $Y$ on $(\boldsymbol{\Omega}, \mathcal{B}, P)$ and $D$ is called a generalized Brownian motion process if $Y(0, \omega)=0$ almost everywhere and for $0=t_{0}<t_{1}<\cdots<$ $t_{n} \leq T$, the $n$-dimensional random vector $\left(Y\left(t_{1}, \omega\right), \ldots, Y\left(t_{n}, \omega\right)\right)$ is normally distributed with density function

$$
\begin{aligned}
W_{n}(\vec{t}, \vec{\eta})= & \left((2 \pi)^{n} \prod_{j=1}^{n}\left(b\left(t_{j}\right)-b\left(t_{j-1}\right)\right)\right)^{-1 / 2} \\
& \cdot \exp \left\{-\frac{1}{2} \sum_{j=1}^{n} \frac{\left(\left(\eta_{j}-a\left(t_{j}\right)\right)-\left(\eta_{j-1}-a\left(t_{j-1}\right)\right)\right)^{2}}{b\left(t_{j}\right)-b\left(t_{j-1}\right)}\right\},
\end{aligned}
$$

where $\vec{\eta}=\left(\eta_{1}, \ldots, \eta_{n}\right), \eta_{0}=0, \vec{t}=\left(t_{1}, \ldots, t_{n}\right), a(t)$ is an absolutely continuous real-valued function on $[0, T]$ with $a(0)=0, a^{\prime}(t) \in L_{2}[0, T]$ and $b(t)$ is a strictly increasing continuously differentiable real-valued function with $b(t)>0$ and $b^{\prime}(t)>0$ for each $t \in[0, T]$.

As explained in [15, pp. 18-20], $Y$ induces a probability measure $\mu$ on the measurable space $\left(\mathbb{R}^{D}, \mathcal{B}^{D}\right)$, where $\mathbb{R}^{D}$ is the space of all real valued functions $x(t), \quad t \in D$, and $\mathcal{B}^{D}$ is the smallest $\sigma$-algebra of subsets of $\mathbb{R}^{D}$ with respect to which all the coordinate evaluation maps $e_{t}(x)=x(t)$ defined on $\mathbb{R}^{D}$ are measurable. The triple $\left(\mathbb{R}^{D}, \mathcal{B}^{D}, \mu\right)$ is a probability measure space. This measure space is called the function space induced by the generalized Brownian motion process $Y$ determined by $a(\cdot)$ and $b(\cdot)$.

We note that the generalized Brownian motion process $Y$ determined by $a(\cdot)$ and $b(\cdot)$ is a Gaussian process with mean function $a(t)$ and covariance function $r(s, t)=\min \{b(s), b(t)\}$. By Theorem $14.2[15$, p. 187], the probability measure $\mu$ induced by $Y$, taking a separable version, is supported by $C_{a, b}[0, T]$ (which is equivalent to the Banach space of continuous functions $x$ on $[0, T]$ with $x(0)=0$ under the sup norm). Hence $\left(C_{a, b}[0, T], \mathcal{B}\left(C_{a, b}[0, T]\right), \mu\right)$ is the function space induced by $Y$, where $\mathcal{B}\left(C_{a, b}[0, T]\right)$ is the Boreal $\sigma$-algebra of $C_{a, b}[0, T]$.

A subset $B$ of $C_{a, b}[0, T]$ is said to be scale-invariant measurable provided $\rho B$ is $\mathcal{B}\left(C_{a, b}[0, T]\right)$-measurable for all $\rho>0$, and a scale-invariant measurable set $N$ is said to be a scale-invariant null set provided $\mu(\rho N)=0$ for all $\rho>0$. A property that holds except on a scale-invariant null set is said to hold scaleinvariant almost everywhere(s-a.e.). If two functionals $F$ and $G$ are equal scale-invariant almost everywhere, we write $F \approx G$.

Let $L_{a, b}^{2}[0, T]$ be the Hilbert space of functions on $[0, T]$ which are Lebesgue measurable and square integrable with respect to the Lebesgue Stieltjes measures on $[0, T]$ induced by $a(\cdot)$ and $b(\cdot)$; i.e.,

$$
L_{a, b}^{2}[0, T]=\left\{v: \int_{0}^{T} v^{2}(s) d b(s)<\infty \text { and } \int_{0}^{T} v^{2}(s) d|a|(s)<\infty\right\},
$$

where $|a|(t)$ denotes the total variation of the function $a$ on the interval $[0, t]$. 
For $u, v \in L_{a, b}^{2}[0, T]$, let

$$
(u, v)_{a, b}=\int_{0}^{T} u(t) v(t) d[b(t)+|a|(t)] .
$$

Then $(\cdot, \cdot)_{a, b}$ is an inner product on $L_{a, b}^{2}[0, T]$ and $\|u\|_{a, b}=\sqrt{(u, u)_{a, b}}$ is a norm on $L_{a, b}^{2}[0, T]$. In particular, note that $\|u\|_{a, b}=0$ if and only if $u(t)=0$ a.e. on $[0, T]$. Furthermore $\left(L_{a, b}^{2}[0, T],\|\cdot\|_{a, b}\right)$ is a separable Hilbert space. Note that all functions of bounded variation on $[0, T]$ are elements of $L_{a, b}^{2}[0, T]$. Also note that if $a(t) \equiv 0$ and $b(t)=t$ on $[0, T]$, then $L_{a, b}^{2}[0, T]=L^{2}[0, T]$. In fact,

$$
\left(L_{a, b}^{2}[0, T],\|\cdot\|_{a, b}\right) \subset\left(L_{0, b}^{2}[0, T],\|\cdot\|_{0, b}\right)=\left(L^{2}[0, T],\|\cdot\|_{2}\right)
$$

since the two norms $\|\cdot\|_{0, b}$ and $\|\cdot\|_{2}$ are equivalent.

Let $\left\{\phi_{j}\right\}_{j=1}^{\infty}$ be a complete orthonormal set of real-valued functions of bounded variation on $[0, T]$ such that

$$
\left(\phi_{j}, \phi_{k}\right)_{a, b}= \begin{cases}0, & j \neq k \\ 1, & j=k,\end{cases}
$$

and for each $v \in L_{a, b}^{2}[0, T]$, let

$$
v_{n}(t)=\sum_{j=1}^{n}\left(v, \phi_{j}\right)_{a, b} \phi_{j}(t)
$$

for $n=1,2, \ldots$. Then for each $v \in L_{a, b}^{2}[0, T]$, the Palely-Wiener-Zygmund (PWZ) stochastic integral $\langle v, x\rangle$ is defined by the formula

$$
\langle v, x\rangle=\lim _{n \rightarrow \infty} \int_{0}^{T} v_{n}(t) d x(t)
$$

for all $x \in C_{a, b}[0, T]$ for which the limit exists; one can show that for each $v \in L_{a, b}^{2}[0, T]$, the PWZ integral $\langle v, x\rangle$ exists for $\mu$-a.e. $x \in C_{a, b}[0, T]$.

Followings are some facts about the PWZ stochastic integral.

(1) For each $v \in L_{a, b}^{2}[0, T]$, the PWZ integral $\langle v, x\rangle$ exists for $\mu$-a.e. $x \in$ $C_{a, b}[0, T]$.

(2) The PWZ integral $\langle v, x\rangle$ is essentially independent of the complete orthonormal set $\left\{\phi_{j}\right\}_{j=1}^{\infty}$.

(3) If $v$ is of bounded variation on $[0, T]$, then the $\mathrm{PWZ}$ integral $\langle v, x\rangle$ equals the Riemann-Stieltjes integral $\int_{0}^{T} v(t) d x(t)$ for s-a.e. $x \in C_{a, b}[0, T]$.

(4) The PWZ integral has the expected linearity properties.

(5) For all $v \in L_{a, b}^{2}[0, T],\langle v, x\rangle$ is a Gaussian random variable with mean $\int_{0}^{T} v(s) d a(s)$ and variance $\int_{0}^{T} v^{2}(s) d b(s)$. 
We denote the function space integral of a $\mathcal{B}\left(C_{a, b}[0, T]\right)$-measurable functional $F$ by

$$
E[F]=\int_{C_{a, b}[0, T]} F(x) d \mu(x)
$$

whenever the integral exists.

\section{Complete orthonormal sets in $L_{2}(\mathbb{R})$ and $L_{2}\left(\mathbb{R}^{n}\right)$}

In this section, we define generalized normalizing Hermite functions. We use them to obtain a complete orthonormal set in $L_{2}(\mathbb{R})$ and $L_{2}\left(\mathbb{R}^{n}\right)$.

Definition 3.1. For each $m=0,1, \ldots$ and $t \in[0, T]$, we define a generalized Hermite polynomial in $\frac{u-a(t)}{\sqrt{b(t)}}$ of degree $m$ by

$$
H_{m}(u ; t) \equiv(-1)^{m}(b(t))^{\frac{m}{2}} \exp \left\{\frac{(u-a(t))^{2}}{2 b(t)}\right\} \frac{d^{m}}{d u^{m}}\left(\exp \left\{-\frac{(u-a(t))^{2}}{2 b(t)}\right\}\right) .
$$

For examples, we see that

$$
H_{0}(u ; t)=1, H_{1}(u ; t)=\frac{u-a(t)}{\sqrt{b(t)}} \text { and } H_{2}(u ; t)=-1+\left(\frac{u-a(t)}{\sqrt{b(t)}}\right)^{2} .
$$

We note that for each $m=0,1, \ldots$ and $t \in[0, T]$,

$$
H_{m+1}(u ; t)=\left(\frac{u-a(t)}{\sqrt{b(t)}}\right) H_{m}(u ; t)-(b(t))^{\frac{1}{2}} H_{m}^{\prime}(u ; t),
$$

where / means $\frac{d}{d u}$. And for each $m=1,2, \ldots$ and $t \in[0, T]$,

$$
H_{m+1}(u ; t)-\left(\frac{u-a(t)}{\sqrt{b(t)}}\right) H_{m}(u ; t)+m H_{m-1}(u ; t)=0
$$

and so

$$
H_{m}^{\prime}(u ; t)=\frac{m}{\sqrt{b(t)}} H_{m-1}(u ; t)
$$

Lemma 3.2. For any nonnegative integers $m$ and $k$,

$$
\begin{aligned}
I & \equiv \int_{\mathbb{R}} \exp \left\{-\frac{(u-a(t))^{2}}{2 b(t)}\right\} H_{k}(u ; t) H_{m}(u ; t) d u \\
& = \begin{cases}0 & \text { if } \quad k \neq m \\
k ! \sqrt{2 \pi b(t)} & \text { if } \quad k=m .\end{cases}
\end{aligned}
$$

Proof. Assume that $m \leq k$. Let

$$
\varphi(u ; t)=\exp \left\{-\frac{(u-a(t))^{2}}{2 b(t)}\right\} .
$$


Then

$$
\exp \left\{-\frac{(u-a(t))^{2}}{2 b(t)}\right\} H_{k}(u ; t)=(-1)^{k}(b(t))^{\frac{k}{2}} \varphi^{(k)}(u ; t) .
$$

By using this above, we obtain that

$$
I \equiv(-1)^{k}(b(t))^{\frac{k}{2}} \int_{\mathbb{R}} \varphi^{(k)}(u ; t) H_{m}(u ; t) d u .
$$

By using integration by parts formulas, we obtain that

$$
\begin{aligned}
I & \equiv(-1)^{k}(b(t))^{\frac{k}{2}}\left[\left.\varphi^{(k-1)}(u ; t) H_{m}(u ; t)\right|_{-\infty} ^{\infty}-\int_{\mathbb{R}} \varphi^{(k-1)}(u ; t) H_{m}^{\prime}(u ; t) d u\right] \\
& =(-1)^{k+1}(b(t))^{\frac{k}{2}} \int_{\mathbb{R}} \varphi^{(k-1)}(u ; t) H_{m}^{\prime}(u ; t) d u .
\end{aligned}
$$

Continuing on this manner, we obtain that

$$
I=(-1)^{k+m}(b(t))^{\frac{k}{2}} \int_{\mathbb{R}} \varphi^{(k-m)}(u ; t) H_{m}^{(m)}(u ; t) d u .
$$

But if $m<k$, then $I=0$. If $k=m$, then by using equation (3.2) above,

$$
\begin{aligned}
I & =(-1)^{2 k}(b(t))^{\frac{k}{2}} \int_{\mathbb{R}} \frac{k !}{b(t)^{\frac{k}{2}}} \exp \left\{-\frac{(u-a(t))^{2}}{2 b(t)}\right\} d u \\
& =k ! \int_{\mathbb{R}} \exp \left\{-\frac{(u-a(t))^{2}}{2 b(t)}\right\} d u \\
& =k ! \sqrt{2 \pi b(t)}
\end{aligned}
$$

which completes the proof of Lemma 3.2.

We are ready to define generalized Hermite functions and a sequence of generalized normalizing Hermite functions $\left\{K_{m}\right\}_{m=0}^{\infty}$ which is a complete orthonormal set in $L_{2}(\mathbb{R})$.

Definition 3.3. For each $m=0,1, \ldots$ and $t \in[0, T]$, we define a generalized Hermite function of degree $m$ by

$$
h_{m}(u ; t) \equiv H_{m}(u ; t) \exp \left\{-\frac{(u-a(t))^{2}}{4 b(t)}\right\}
$$

and we define a generalized normalizing Hermite function of degree $m$ by

$$
K_{m}(u ; t) \equiv(m ! \sqrt{2 \pi b(t)})^{-\frac{1}{2}} h_{m}(u ; t) .
$$

Now, we are ready to obtain a complete orthonormal set in $L_{2}(\mathbb{R})$.

Theorem 3.4. The set of functions $\left\{K_{m}\right\}_{m=0}^{\infty}$ defined by $(3.4)$ is an orthonormal set in $L_{2}(\mathbb{R})$. 
Proof. By using equation (3.3) above, it immediately follows that for all nonnegative integers $k$ and $m$,

$$
\begin{aligned}
& \int_{\mathbb{R}} K_{k}(u ; t) K_{m}(u ; t) d u \\
= & \int_{\mathbb{R}}(k ! \sqrt{2 \pi b(t)})^{-\frac{1}{2}} h_{k}(u ; t)(m ! \sqrt{2 \pi b(t)})^{-\frac{1}{2}} h_{m}(u ; t) d u \\
= & (k ! \sqrt{2 \pi b(t)})^{-\frac{1}{2}}(m ! \sqrt{2 \pi b(t)})^{-\frac{1}{2}} \int_{\mathbb{R}} H_{k}(u ; t) H_{m}(u ; t) \exp \left\{-\frac{(u-a(t))^{2}}{2 b(t)}\right\} d u \\
= & \delta_{k, m} .
\end{aligned}
$$

Theorem 3.5. The set $\left\{K_{m}\right\}_{m=0}^{\infty}$ is a complete orthonormal set in $L_{2}(\mathbb{R})$.

Proof. For each $m=0,1, \ldots$ and $f \in L_{2}(\mathbb{R})$, assume that

$$
\int_{\mathbb{R}} K_{m}(u ; t) f(u) d u=0 .
$$

It suffices to show that $f=0$ a.e.. Let

$$
g(u)=\exp \left\{-\frac{(u-a(t))^{2}}{4 b(t)}\right\} f(u)
$$

for $u \in \mathbb{R}$. Then $g \in L_{1}(\mathbb{R})$ and so the Fourier transform

$$
F(z) \equiv \int_{\mathbb{R}} \exp \{i z u\} g(u) d u
$$

exists for all $z \in \mathbb{C}$. Also $\left.\frac{1}{\sqrt{2 \pi}} F\right|_{\mathbb{R}}$ is the inverse Fourier transform of $g$. Thus if $F(z) \equiv 0$, then by uniqueness of the inverse Fourier transform, $g=0$ and so $f=0$ a.e. on $\mathbb{R}$.

Since $F$ is an entire function, we can write

$$
F(z)=\sum_{n=0}^{\infty} b_{n} z^{n}
$$

where $b_{n}=\frac{F^{(n)}(0)}{n !}$. But

$$
F^{(n)}(z)=\int_{\mathbb{R}}(i u)^{n} \exp \left\{i z u-\frac{(u-a(t))^{2}}{4 b(t)}\right\} f(u) d u
$$

and $F^{(n)}(0)=0$ and hence $b_{n}=0$ for all $n$ and so $F(z)=0$.

Definition 3.6. For each $j=1,2, \ldots$, let $m_{j}$ be a nonnegative integer. For $\left(u_{1}, \ldots, u_{n}\right) \in \mathbb{R}^{n}$, let

$$
K_{\left(m_{1}, \ldots, m_{n}\right)}\left(u_{1}, \ldots, u_{n} ; t\right) \equiv \prod_{j=1}^{n} K_{m_{j}}\left(u_{j} ; t\right),
$$

where $K_{m_{j}}\left(u_{j} ; t\right)$ is given by equation (3.4) above. 
In our next theorem, we also give a complete orthonormal set in $L_{2}\left(\mathbb{R}^{n}\right)$.

Theorem 3.7. The set of generalized normalizing Hermite functions

$$
\left\{K_{\left(m_{1}, \ldots, m_{n}\right)}\right\}_{m_{1}, \ldots, m_{n}=0}^{\infty}
$$

is a complete orthonormal set in $L_{2}\left(\mathbb{R}^{n}\right)$.

Proof. By using Theorem 3.4 and the fact that the set of functions of the form $\left\{f_{1} f_{2} \cdots f_{n}: f_{j} \in L_{2}(\mathbb{R}), j=1, \ldots, n\right\}$ is dense in $L_{2}\left(\mathbb{R}^{n}\right)$, we obtain the desired result.

Definition 3.8. For each $g \in L_{2}\left(\mathbb{R}^{n}\right)$, the generalized Hermite coefficient of $g$ with respect to the complete orthonormal set $\left\{K_{\left(m_{1}, \ldots, m_{n}\right)}\right\}_{m_{1}, \ldots, m_{n}=0}^{\infty}$ is defined by the formula

$$
\begin{aligned}
a_{\left(m_{1}, \ldots, m_{n}\right)}^{g} & \equiv \int_{\mathbb{R}^{n}} g(\vec{u}) K_{\left(m_{1}, \ldots, m_{n}\right)}(\vec{u} ; t) d \vec{u} \\
& =\int_{\mathbb{R}^{n}} g(\vec{u})\left(\prod_{j=1}^{n} K_{m_{j}}\left(u_{j} ; t\right)\right) d \vec{u} .
\end{aligned}
$$

Remark 3.9. By Theorem 3.7, it follows that

$$
g\left(u_{1}, \ldots, u_{n}\right)=\lim _{N \rightarrow \infty} \sum_{m_{1}, \ldots, m_{n}=0}^{N} a_{\left(m_{1}, \ldots, m_{n}\right)}^{g} \prod_{j=1}^{n} K_{m_{j}}\left(u_{j} ; t\right) ;
$$

that is to say that

$$
\int_{\mathbb{R}^{n}}\left|g(\vec{u})-\sum_{m_{1}, \ldots, m_{n}=0}^{N} a_{\left(m_{1}, \ldots, m_{n}\right)}^{g} \prod_{j=1}^{n} K_{m_{j}}\left(u_{j} ; t\right)\right|^{2} d \vec{u}
$$

goes to zero as $N \rightarrow \infty$.

\section{A complete orthonormal set in $L_{2}\left(C_{a, b}[0, T]\right)$}

In this section, we define the generalized Fourier-Hermite coefficient and the generalized Fourier-Hermite functionals. We then obtain a complete orthonormal set in $L_{2}\left(C_{a, b}[0, T]\right)$.

The following notations are used throughout this paper:

$$
A_{j} \equiv \int_{0}^{T} \alpha_{j}(t) d a(t)
$$

and

$$
B_{j} \equiv \int_{0}^{T} \alpha_{j}^{2}(t) d b(t)
$$


where $\left\{\alpha_{j}\right\}$ is a complete orthonormal set in $L_{a, b}^{2}[0, T]$. We note that for each $j=1,2, \ldots$,

$$
0<B_{j}=\int_{0}^{T} \alpha_{j}^{2}(t) d b(t) \leq \int_{0}^{T} \alpha_{j}^{2}(t) d[b(t)+|a|(t)]=\left\|\alpha_{j}\right\|_{a, b}^{2}=1,
$$

while $A_{j}$ may be positive, negative or zero. We also note that if $a(t) \equiv 0$ on $[0, T]$, then $A_{j}=0$ and $B_{j}=1$ for each $j=1,2, \ldots$

The following integration formula used several times throughout this paper:

Let $\left\{\alpha_{1}, \ldots, \alpha_{n}\right\}$ be an orthonormal set of functions from $\left(L_{a, b}^{2}[0, T],\|\cdot\|_{a, b}\right)$. Let $h: \mathbb{R}^{n} \rightarrow \mathbb{R}$ be Lebesgue measurable and let $H(x)=h\left(\left\langle\alpha_{1}, x\right\rangle, \ldots,\left\langle\alpha_{n}, x\right\rangle\right)$. Then

$$
\begin{aligned}
\int_{C_{a, b}[0, T]} H(x) d \mu(x)= & \left(\prod_{j=1}^{n} 2 \pi B_{j}\right)^{-\frac{1}{2}} \int_{\mathbb{R}^{n}} h\left(u_{1}, \ldots, u_{n}\right) \\
& \cdot \exp \left\{-\sum_{j=1}^{n} \frac{\left(u_{j}-A_{j}\right)^{2}}{2 B_{j}}\right\} d u_{1} \cdots d u_{n}
\end{aligned}
$$

in the sense that if either side exists, both sides exist and equality holds.

Using formula (4.3) we observe that $E\left[\left\langle\alpha_{j}, x\right\rangle\right]=A_{j}, E\left[\left\langle\alpha_{j}, x\right\rangle^{2}\right]=B_{j}+A_{j}^{2}$ and that $\operatorname{Var}\left(\left\langle\alpha_{j}, x\right\rangle\right)=B_{j}$ for each $j=1, \ldots, n$.

Also note that the complete orthonormal set $\left\{\alpha_{1}, \alpha_{2}, \ldots\right\}$ in $L_{a, b}^{2}[0, T]$ is completely at our disposal. For example, we could choose the $\alpha_{j}$ 's to the continuous and of bounded variation on $[0, T]$, or we could choose the $\alpha_{j}$ 's to the Haar functions on $[0, T]$, etc.

To obtain a complete orthonormal set in $L_{2}\left(C_{a, b}[0, T]\right)$, we define a generalized Hermite polynomial and a generalized normalizing Hermite function as below.

Definition 4.1. For each $m=0,1, \ldots$ and for each $j=1,2, \ldots$, we define a generalized Hermite polynomial by

$$
\tilde{H}_{m}^{j}(u) \equiv(-1)^{m}(m !)^{-\frac{1}{2}}\left(B_{j}\right)^{\frac{m}{2}} \exp \left\{\frac{\left(u-A_{j}\right)^{2}}{2 B_{j}}\right\} \frac{d^{m}}{d u^{m}}\left(\exp \left\{-\frac{\left(u-A_{j}\right)^{2}}{2 B_{j}}\right\}\right)
$$

and we define a generalized normalizing Hermite polynomial by

$$
\tilde{K}_{m}^{j}(u) \equiv\left(2 \pi B_{j}\right)^{-\frac{1}{4}} \tilde{H}_{m}^{j}(u) \exp \left\{-\frac{\left(u-A_{j}\right)^{2}}{4 B_{j}}\right\},
$$

where $A_{j}$ and $B_{j}$ are as in equations (4.1) and (4.2) above respectively.

Remark 4.2. (1) If $a(t) \equiv 0$ on $[0, T]$, then for all $j=1,2, \ldots, A_{j}=0$ and $B_{j}=1$. In case, $\tilde{H}_{m}^{j}$ and $\tilde{K}_{m}^{j}$ are defined independently of $j$ 's. 
(2) We can easily see that for each $j=1,2, \ldots,\left\{\tilde{K}_{m}^{j}\right\}_{m=0}^{\infty}$ is a complete orthonormal set in $L_{2}(\mathbb{R})$. Furthermore, for all $j=1, \ldots, n$, let $m_{j}$ be a nonnegative integer. Then the set $\left\{\tilde{K}_{\left(m_{1}, \ldots, m_{n}\right)}^{(1, \ldots, n)}\right\}_{m_{1}, \ldots, m_{n}=0}^{\infty}$ is a complete orthonormal set in $L_{2}\left(\mathbb{R}^{n}\right)$, where $\tilde{K}_{\left(m_{1}, \ldots, m_{n}\right)}^{(1, \ldots, n)}(\vec{u})=\prod_{j=1}^{n} \tilde{K}_{m_{j}}^{j}\left(u_{j}\right)$.

(3) For each positive integer $k$ and nonnegative integer $m$, let

$$
\varphi_{(m, k)}(x) \equiv \tilde{H}_{m}^{k}\left(\left\langle\alpha_{k}, x\right\rangle\right) .
$$

For each $j=1, \ldots, k$, let $m_{j}$ be a nonnegative integer. Let

$$
\begin{aligned}
\Phi_{\left(m_{1}, \ldots, m_{k}\right)}(x) & \equiv \varphi_{\left(m_{1}, 1\right)}(x) \varphi_{\left(m_{2}, 2\right)}(x) \cdots \varphi_{\left(m_{k}, k\right)}(x) \\
& =\prod_{j=1}^{k} \tilde{H}_{m_{j}}^{j}\left(\left\langle\alpha_{k}, x\right\rangle\right) .
\end{aligned}
$$

The functionals in equation (4.4) are called the generalized Fourier-Hermite functionals on $C_{a, b}[0, T]$.

The three assertions in the following statements follow by easily from Remark 4.2:

(1) $\varphi_{(0, k)}(x) \equiv 1$ for all positive integer $k$.

(2) $\Phi_{\left(m_{1}, \ldots, m_{k}\right)}(x)=\Phi_{\left(m_{1}, \ldots, m_{k}, 0, \ldots, 0\right)}(x)$ for all positive integer $k$.

(3) Inserting zeroes to the left of a non-zero entry in $\Phi$ change the values of $\Phi$.

Theorem 4.3. The set of functionals $\mathcal{M} \equiv\left\{\Phi_{\left(m_{1}, \ldots, m_{k}\right)}\right\}_{k=1}^{\infty}$ is an orthonormal set in $L_{2}\left(C_{a, b}[0, T]\right)$.

Proof. By using formula (4.3) above, it follows that

$$
\begin{aligned}
& \int_{C_{a, b}[0, T]} \Phi_{\left(m_{1}, \ldots, m_{k}\right)}(x) \Phi_{\left(n_{1}, \ldots, n_{k}\right)}(x) d \mu(x) \\
= & \int_{C_{a, b}[0, T]}\left[\prod_{j=1}^{k} \tilde{H}_{m_{j}}^{j}\left(\left\langle\alpha_{j}, x\right\rangle\right)\right]\left[\prod_{j=1}^{k} \tilde{H}_{n_{j}}^{j}\left(\left\langle\alpha_{j}, x\right\rangle\right)\right] d \mu(x) \\
= & \left(\prod_{j=1}^{k} 2 \pi B_{j}\right)^{-\frac{1}{2}} \int_{\mathbb{R}^{k}}\left[\prod_{j=1}^{k} \tilde{H}_{m_{j}}^{j}\left(u_{j}\right)\right]\left[\prod_{j=1}^{k} \tilde{H}_{n_{j}}^{j}\left(u_{j}\right)\right] \exp \left\{-\sum_{j=1}^{k} \frac{\left(u_{j}-A_{j}\right)^{2}}{2 B_{j}}\right\} d u_{1} \cdots d u_{k} \\
= & \int_{\mathbb{R}^{k}}\left[\prod_{j=1}^{k} \tilde{K}_{m_{j}}^{j}\left(u_{j}\right)\right]\left[\prod_{j=1}^{k} \tilde{K}_{n_{j}}^{j}\left(u_{j}\right)\right] d u_{1} \cdots d u_{k} \\
= & \delta_{m_{1}, n_{1}} \delta_{m_{2}, n_{2}} \cdots \delta_{m_{k}, n_{k}} .
\end{aligned}
$$

In order to show that the set of functionals $\mathcal{M}$ in Theorem 4.3 is complete we will show that every functional $F$ in $L_{2}\left(C_{a, b}[0, T]\right)$ has a generalized FourierHermite series expansion in terms of the functionals in $\mathcal{M}$. 
Definition 4.4. For each $F \in L_{2}\left(C_{a, b}[0, T]\right)$ and $\Phi_{\left(m_{1}, \ldots, m_{k}\right)} \in \mathcal{M}$, the generalized Fourier-Hermite coefficient of $F$ is defined by

$$
A_{\left(m_{1}, \ldots, m_{k}\right)}^{F} \equiv \int_{C_{a, b}[0, T]} F(x) \Phi_{\left(m_{1}, \ldots, m_{k}\right)}(x) d \mu(x) .
$$

Lemma 4.5. For $\vec{u}=\left(u_{1}, \ldots, u_{n}\right) \in \mathbb{R}^{n}$, let $f(\vec{u})$ be a measurable function such that

$$
f(\vec{u}) \exp \left\{-\sum_{j=1}^{n} \frac{\left(u_{j}-A_{j}\right)^{2}}{4 B_{j}}\right\} \in L_{2}\left(\mathbb{R}^{n}\right) .
$$

Let $F: C_{a, b}[0, T] \rightarrow \mathbb{R}$ be a functional defined by

$$
F(x)=f\left(\left\langle\alpha_{1}, x\right\rangle, \ldots,\left\langle\alpha_{n}, x\right\rangle\right),
$$

where $\left\{\alpha_{j}\right\}$ is a complete orthonormal set in $L_{a, b}^{2}[0, T]$. Then

$$
I \equiv \int_{C_{a, b}[0, T]}\left|F(x)-\sum_{m_{1}, \ldots, m_{n}=0}^{N} A_{\left(m_{1}, \ldots, m_{n}\right)}^{F} \Phi_{\left(m_{1}, \ldots, m_{n}\right)}(x)\right|^{2} d \mu(x) \rightarrow 0
$$

as $N \rightarrow \infty$.

Theorem 4.6. Let $\mathcal{L}$ be the set of all functionals $F$ which has the form (4.6) above. Then $\mathcal{L}$ is dense in $L_{2}\left(C_{a, b}[0, T]\right)$.

Proof. By the usual Lebesgue argument, it suffices to show that the characteristic functional can be represented by linear combination of elements of $\mathcal{L}$. For $0=t_{0}<t_{1}<\cdots<t_{n} \leq T$, let $I=\left\{x \in C_{a, b}[0, T]: \alpha_{j} \leq x\left(t_{j}\right) \leq \beta_{j}\right\}$. Then

$$
\chi_{I}(x)=\prod_{j=1}^{n} \chi_{\left[\alpha_{j}, \beta_{j}\right]}\left(x\left(t_{j}\right)\right) \text {. }
$$

For given $\varepsilon>0$ and $j=1, \ldots, n$, define a trapezoid function $Z_{j, \epsilon}$ by

$$
Z_{j, \varepsilon}(s)=\left\{\begin{array}{cl}
0 & \text { if } s \in\left(-\infty, \alpha_{j}-\varepsilon\right) \\
\frac{1}{\varepsilon}\left(s-\alpha_{j}\right)+1 & \text { if } s \in\left[\alpha_{j}-\varepsilon, \alpha_{j}\right) \\
1 & \text { if } s \in\left[\alpha_{j}, \beta_{j}\right] \\
-\frac{1}{\varepsilon}\left(s-\beta_{j}\right)+1 & \text { if } s \in\left(\beta_{j}, \beta_{j}+\varepsilon\right] \\
0 & \text { if } s \in\left(\beta_{j}+\varepsilon, \infty\right) .
\end{array}\right.
$$

Let $C_{\varepsilon}(x) \equiv \prod_{j=1}^{n} Z_{j, \varepsilon}\left(x\left(t_{j}\right)\right)$. Then $C_{\varepsilon}(x) \rightarrow \chi_{I}(x)$ as $\varepsilon \downarrow 0$. Next, let

$$
g_{j}(t) \equiv \chi_{\left[0, t_{j}\right]}(t) \text {. }
$$

Then we write that $g_{j}(t) \equiv \sum_{l=1}^{\infty} b_{j, l} \alpha_{l}(t)$. Let $G_{j, m}(t) \equiv \sum_{l=1}^{m} b_{j, l} \alpha_{l}(t)$. Then

$$
\left\|g_{j}-G_{j, m}\right\|_{a, b} \rightarrow 0 \text { as } m \rightarrow \infty \text {. }
$$

Thus we obtain that

$$
\left\langle G_{j, m}, x\right\rangle \rightarrow\left\langle g_{j}, x\right\rangle
$$


in the $L_{2}\left(C_{a, b}[0, T]\right)$ sense as $m \rightarrow \infty$. Thus there exists a subsequence $\left\{G_{j, m_{k}}\right\}$ of $\left\{G_{j, m}\right\}$ such that

$$
\left\langle G_{j, m_{k}}, x\right\rangle \rightarrow\left\langle g_{j}, x\right\rangle
$$

as $k \rightarrow \infty$ for a.e. $x \in C_{a, b}[0, T]$. Let $S_{\varepsilon, k}(x) \equiv \prod_{j=1}^{n} Z_{j, \varepsilon}\left(\left\langle G_{j, m_{k}}, x\right\rangle\right)$. Then

$$
S_{\varepsilon, k}(x) \rightarrow \prod_{j=1}^{n} Z_{j, \varepsilon}\left(\left\langle g_{j}, x\right\rangle\right)
$$

as $k \rightarrow \infty$ for a.e. $x \in C_{a, b}[0, T]$. Thus $S_{\varepsilon, k}(x) \rightarrow C_{\varepsilon}(x)$ in the $L_{2}\left(C_{a, b}[0, T]\right)$ sense as $k \rightarrow \infty$. But $S_{\varepsilon, k}(x)$ is an element of $\mathcal{L}$. Hence we complete the proof as desired.

Remark 4.7. Note, we have shown above for $F$ given by equation (4.6), that $A_{\left(m_{1}, \ldots, m_{k}\right)}$ given by (4.5) is zero for $k>n$ and $m_{k}>0$. Hence the number of subscripts on $\Phi$ and $A^{F}$ in (4.7) may be increased beyond $n$ without changing the sum. Hence we may take $N>n$ subscripts in (4.7) and thus for any $F$ given by (4.6),

$$
\int_{C_{a, b}[0, T]}\left|F(x)-\sum_{m_{1}, \ldots, m_{N}=0}^{N} A_{\left(m_{1}, \ldots, m_{N}\right)}^{F} \Phi_{\left(m_{1}, \ldots, m_{N}\right)}(x)\right|^{2} d \mu(x) \rightarrow 0
$$

as $N \rightarrow \infty$. That is to say,

$$
F(x)=\lim _{N \rightarrow \infty} \sum_{m_{1}, \ldots, m_{N}=0}^{N} A_{\left(m_{1}, \ldots, m_{N}\right)}^{F} \Phi_{\left(m_{1}, \ldots, m_{N}\right)}(x),
$$

and the right-hand side of (4.8) is called the generalized Fourier-Hermite series of $F$.

Theorem 4.6 above, together with Remark 4.7, tell us that every $F \in$ $L_{2}\left(C_{a, b}[0, T]\right)$ has a convergent generalized Fourier-Hermite series expansion. This observation plays a key role in Section 5 below.

Corollary 4.8. Let $F$ be an any functional on $C_{a, b}[0, T]$ with

$$
\int_{C_{a, b}[0, T]}|F(x)|^{2} d \mu(x)<\infty
$$

and for $N=1,2, \ldots$, let

$$
F_{N}(x)=\sum_{m_{1}, \ldots, m_{N}=0}^{N} A_{\left(m_{1}, \ldots, m_{N}\right)}^{F} \Phi_{\left(m_{1}, \ldots, m_{N}\right)}(x) .
$$

Then

$$
\int_{C_{a, b}[0, T]}\left|F_{N}(x)-F(x)\right|^{2} d \mu(x) \rightarrow 0
$$


as $N \rightarrow \infty$ and

$$
\begin{aligned}
F(x) & =\lim _{N \rightarrow \infty} F_{N}(x) \\
& =\lim _{N \rightarrow \infty} \sum_{m_{1}, \ldots, m_{N}=0}^{N} A_{\left(m_{1}, \ldots, m_{N}\right)}^{F} \Phi_{\left(m_{1}, \ldots, m_{N}\right)}(x)
\end{aligned}
$$

is called the generalized Fourier-Hermite series expansion of F.

\section{The generalized Fourier-Wiener function space transforms}

In this section we obtain a formula for the generalized Fourier-Wiener function space transform of the generalized Fourier-Hermite function. We then proceed to obtain formula for the generalized Fourier-Wiener function space transform of a functional $F$ in $L_{2}\left(C_{a, b}[0, T]\right)$.

Let $K_{a, b}[0, T]$ be the set of all complex-valued continuous functions $x(t)$ defined on $[0, T]$ which vanish at $t=0$ and whose real and imaginary parts are elements of $C_{a, b}[0, T]$; namely,

$$
K_{a, b}[0, T]=\left\{x:[0, T] \rightarrow \mathbb{C} \mid x(0)=0, \operatorname{Re}(x), \operatorname{Im}(x) \in C_{a, b}[0, T]\right\} .
$$

Then $C_{a, b}[0, T]$ is a subspace of all real-valued functions in $K_{a, b}[0, T]$.

Definition 5.1. Let $F$ be a functional defined on $K_{a, b}[0, T]$. The generalized Fourier-Wiener function space transform $\mathcal{F}_{\sqrt{2}, i}(F)$ of $F$ is defined by

$$
\mathcal{F}_{\sqrt{2}, i}(F)(y)=\int_{C_{a, b}[0, T]} F(\sqrt{2} x+i y) d \mu(x), \quad y \in K_{a, b}[0, T]
$$

if it exists.

Remark 5.2. When $a(t) \equiv 0$ and $b(t)=t$ on $[0, T]$, the generalized FourierWiener function space transform is the modified Fourier-Wiener transform introduced by Cameron and Martin in [3].

Throughout this paper, in order to ensure that various integrals exist, we will assume that $\beta=c+i d$ is a nonzero complex number satisfying the inequality

$$
\operatorname{Re}\left(1-\beta^{2}\right)=1+d^{2}-c^{2}>0 .
$$

We note that $\operatorname{Re}\left(1-\beta^{2}\right)=1+d^{2}-c^{2}>0$ if and only if the point $(c, d) \in \mathbb{R}^{2}$ lies in the open region determined by the hyperbola $c^{2}-d^{2}=1$ containing the $d$-axis. Hence, for all $|\beta| \leq 1, \beta \neq \pm 1$ and $\operatorname{Re}\left(1-\beta^{2}\right)>0$. Let $\gamma=\sqrt{1-\beta^{2}}$ with $-\frac{\pi}{4}<\arg (\gamma)<\frac{\pi}{4}$ so that $\gamma^{2}+\beta^{2}=1$ and $\operatorname{Re}\left(\gamma^{2}\right)=\operatorname{Re}\left(1-\beta^{2}\right)>0$.

The following lemma plays a key role in finding the generalized integral transform of functionals given by equation (4.4). 
Lemma 5.3. Let $\beta=c+i d$ be a nonzero complex number with $\beta \neq \pm 1$, satisfying inequality (5.2) and let $\gamma=\sqrt{1-\beta^{2}}$. Then for $\lambda \in \mathbb{R}, n=0,1,2, \ldots$, and $j=1,2, \ldots$,

$$
\begin{aligned}
& \int_{\mathbb{R}} \tilde{H}_{n}^{j}(u) \exp \left\{-\frac{1}{2 \gamma^{2} B_{j}}\left(u-\left(\lambda \beta+\gamma A_{j}\right)\right)^{2}\right\} d u \\
= & \beta^{n} \sqrt{2 \pi \gamma^{2} B_{j}} \tilde{H}_{n}^{j}\left(\lambda+\frac{\gamma+\beta-1}{\beta} A_{j}\right) .
\end{aligned}
$$

Proof. Since $\tilde{H}_{n}^{j}(u)$ is a polynomial of degree $n, B_{j}>0$ and $\operatorname{Re}\left(\gamma^{2}\right)>0$, the integral in (5.3) exists and

$$
\begin{aligned}
I_{n} \equiv & \int_{\mathbb{R}} \tilde{H}_{n}^{j}(u) \exp \left\{-\frac{1}{2 \gamma^{2} B_{j}}\left(u-\left(\lambda \beta+\gamma A_{j}\right)\right)^{2}\right\} d u \\
= & (-1)^{n}(n !)^{-\frac{1}{2}} B_{j}^{\frac{n}{2}} \int_{\mathbb{R}} \exp \left\{\frac{\left(u-A_{j}\right)^{2}}{2 B_{j}}\right\} \frac{d^{n}}{d u^{n}}\left(\exp \left\{-\frac{\left(u-A_{j}\right)^{2}}{2 B_{j}}\right\}\right) \\
= & (-1)^{n}(n !)^{-\frac{1}{2}} B_{j}^{\frac{n}{2}} \exp \left\{-\frac{1}{2 \gamma^{2} B_{j}}\left(u-\left(\lambda \beta+\gamma A_{j}\right)\right)^{2}\right\} d u \\
& \cdot \int_{\mathbb{R}} \exp \left\{-\frac{1}{2 \beta^{2} B_{j}}\left(\left(\beta \lambda+\gamma A_{j}\right)-A_{j}\right)^{2}\right\} \\
2 \gamma^{2} B_{j} & \left.\left(u-\frac{\left(\beta \lambda+\gamma A_{j}\right)-\gamma^{2} A_{j}}{\beta^{2}}\right)^{2}\right\} \frac{d^{n}}{d u^{n}}\left(\exp \left\{-\frac{\left(u-A_{j}\right)^{2}}{2 B_{j}}\right\}\right) d u .
\end{aligned}
$$

Next, integrating by parts $n$ times, we obtain that

$$
\begin{aligned}
I_{n}= & (n !)^{-\frac{1}{2}} B_{j}^{\frac{n}{2}} \exp \left\{\frac{1}{2 \beta^{2} B_{j}}\left(\left(\beta \lambda+\gamma A_{j}\right)-A_{j}\right)^{2}\right\} \\
& \cdot \int_{\mathbb{R}} \frac{d^{n}}{d u^{n}}\left(\exp \left\{-\frac{\beta^{2}}{2 \gamma^{2} B_{j}}\left(u-\frac{\left(\beta \lambda+\gamma A_{j}\right)-\gamma^{2} A_{j}}{\beta^{2}}\right)^{2}\right\}\right) \exp \left\{-\frac{\left(u-A_{j}\right)^{2}}{2 B_{j}}\right\} d u \\
= & (n !)^{-\frac{1}{2}} B_{j}^{\frac{n}{2}}(-\beta)^{n} \exp \left\{\frac{1}{2 \beta^{2} B_{j}}\left(\left(\beta \lambda+\gamma A_{j}\right)-A_{j}\right)^{2}\right\} \\
& \cdot \int_{\mathbb{R}} \frac{d^{n}}{d \lambda^{n}}\left(\exp \left\{-\frac{\beta^{2}}{2 \gamma^{2} B_{j}}\left(u-\frac{\left(\beta \lambda+\gamma A_{j}\right)-\gamma^{2} A_{j}}{\beta^{2}}\right)^{2}\right\}\right) \exp \left\{-\frac{\left(u-A_{j}\right)^{2}}{2 B_{j}}\right\} d u \\
= & (n !)^{-\frac{1}{2}} B_{j}^{\frac{n}{2}}(-\beta)^{n} \exp \left\{\frac{1}{2 \beta^{2} B_{j}}\left(\left(\beta \lambda+\gamma A_{j}\right)-A_{j}\right)^{2}\right\} \\
& \cdot \frac{d^{n}}{d \lambda^{n}}\left(\int_{\mathbb{R}} \exp \left\{-\frac{\beta^{2}}{2 \gamma^{2} B_{j}}\left(u-\frac{\left(\beta \lambda+\gamma A_{j}\right)-\gamma^{2} A_{j}}{\beta^{2}}\right)^{2}\right\} \exp \left\{-\frac{\left(u-A_{j}\right)^{2}}{2 B_{j}}\right\} d u\right) \\
= & (n !)^{-\frac{1}{2}} B_{j}^{\frac{n}{2}}(-\beta)^{n} \exp \left\{\frac{1}{2 \beta^{2} B_{j}}\left(\left(\beta \lambda+\gamma A_{j}\right)-A_{j}\right)^{2}\right\} \\
& \cdot \frac{d^{n}}{d \lambda^{n}}\left(\int_{\mathbb{R}} \exp \left\{-\frac{1}{2 \gamma^{2} B_{j}}\left[u-\left(\beta \lambda+\gamma A_{j}\right)\right]^{2}\right\} d u \exp \left\{-\frac{1}{2 \beta^{2} B_{j}}\left[\left(\beta \lambda+\gamma A_{j}\right)-A_{j}\right]^{2}\right\}\right)
\end{aligned}
$$




$$
\begin{aligned}
= & (n !)^{-\frac{1}{2}} B_{j}^{\frac{n}{2}}(-\beta)^{n} \exp \left\{\frac{1}{2 \beta^{2} B_{j}}\left(\left(\beta \lambda+\gamma A_{j}\right)-A_{j}\right)^{2}\right\} \\
& \cdot \frac{d^{n}}{d \lambda^{n}}\left(\gamma \sqrt{2 \pi B_{j}} \exp \left\{-\frac{1}{2 \beta^{2} B_{j}}\left[\left(\beta \lambda+\gamma A_{j}\right)-A_{j}\right]^{2}\right\}\right) \\
= & (n !)^{-\frac{1}{2}} B_{j}^{\frac{n}{2}}(-\beta)^{n} \exp \left\{\frac{1}{2 \beta^{2} B_{j}}\left(\left(\beta \lambda+\gamma A_{j}\right)-A_{j}\right)^{2}\right\} \\
& \cdot \gamma \sqrt{2 \pi B_{j}} \frac{d^{n}}{d \lambda^{n}}\left(\exp \left\{-\frac{1}{2 \beta^{2} B_{j}}\left[\left(\beta \lambda+\gamma A_{j}\right)-A_{j}\right]^{2}\right)\right. \\
= & (n !)^{-\frac{1}{2}} B_{j}^{\frac{n}{2}}(-\beta)^{n} \exp \left\{\frac{1}{2 B_{j}}\left(\left(\lambda+\frac{\gamma}{\beta} A_{j}\right)-\frac{1}{\beta} A_{j}\right)^{2}\right\} \\
& \cdot \gamma \sqrt{2 \pi B_{j}} \frac{d^{n}}{d \lambda^{n}}\left(\exp \left\{-\frac{1}{2 B_{j}}\left[\left(\lambda+\frac{\gamma}{\beta} A_{j}\right)-\frac{1}{\beta} A_{j}\right]^{2}\right)\right. \\
= & \beta^{n} \sqrt{2 \pi \gamma^{2} B_{j}} \tilde{H}_{n}^{j}\left(\lambda+\frac{\gamma+\beta-1}{\beta} A_{j}\right)
\end{aligned}
$$

which is complete the proof of Lemma 5.3.

Remark 5.4. Equation (5.3) actually holds for each $\lambda \in \mathbb{C}$ since $\tilde{H}_{m_{j}}^{j}(u)$ is a polynomial of degree $m_{j}$ in $\frac{u-A_{j}}{\sqrt{B_{j}}}$ and thus both sides of equation (5.3) are analytic functions of $\lambda$ throughout $\mathbb{C}$.

Lemma 5.5. Let $\beta=i$ and $\gamma=\sqrt{2}$. Then $\beta$ and $\gamma$ satisfying the hypothesis of Lemma 5.3. For each positive integer $j$, let $m_{j}$ be a nonnegative integer, and let $\varphi_{\left(m_{j}, j\right)}(x)=\tilde{H}_{m_{j}}^{j}\left(\left\langle\alpha_{j}, x\right\rangle\right)$. Then for each $y \in K_{a, b}[0, T]$, the generalized Fourier-Wiener function space transform $\mathcal{F}_{\sqrt{2}, i}\left(\varphi_{\left(m_{j}, j\right)}\right)(y)$ exists and is given by the formula

$$
\begin{aligned}
\mathcal{F}_{\sqrt{2}, i}\left(\varphi_{\left(m_{j}, j\right)}\right)(y) & =\mathcal{F}_{\sqrt{2}, i}\left(\tilde{H}_{m_{j}}^{j}\right)\left(\left\langle\alpha_{j}, y\right\rangle\right) \\
& =i^{m_{j}} \tilde{H}_{m_{j}}^{j}\left(\left\langle\alpha_{j}, y\right\rangle+\frac{\sqrt{2}+i-1}{i} A_{j}\right) \\
& =i^{m_{j}} \tilde{H}_{m_{j}}^{j}\left(\left\langle\alpha_{j}, y\right\rangle-i(\sqrt{2}+i-1) A_{j}\right) .
\end{aligned}
$$

Proof. Using equation (5.1), formula (4.3), equation (5.3) with $\lambda=\left\langle\alpha_{j}, y\right\rangle$ and $n=m_{j}$, it follows that

$$
\begin{aligned}
& \mathcal{F}_{\sqrt{2}, i}\left(\varphi_{\left(m_{j}, j\right)}\right)(y)=\mathcal{F}_{\sqrt{2}, i}\left(\tilde{H}_{m_{j}}^{j}\right)\left(\left\langle\alpha_{j}, y\right\rangle\right) \\
= & \int_{C_{a, b}[0, T]} \tilde{H}_{m_{j}}^{j}\left(\sqrt{2}\left\langle\alpha_{j}, x\right\rangle+i\left\langle\alpha_{j}, y\right\rangle\right) d \mu(x) \\
= & \left(2 \pi B_{j}\right)^{-\frac{1}{2}} \int_{\mathbb{R}} \tilde{H}_{m_{j}}^{j}\left(\sqrt{2} u_{j}+i\left\langle\alpha_{j}, y\right\rangle\right) \exp \left\{-\frac{\left(u_{j}-A_{j}\right)^{2}}{2 B_{j}}\right\} d u_{j}
\end{aligned}
$$




$$
\begin{aligned}
& =\left(2 \pi B_{j}\right)^{-\frac{1}{2}} \int_{\mathbb{R}} \tilde{H}_{m_{j}}^{j}\left(\gamma u_{j}+\beta\left\langle\alpha_{j}, y\right\rangle\right) \exp \left\{-\frac{\left(u_{j}-A_{j}\right)^{2}}{2 B_{j}}\right\} d u_{j} \\
& =\left(2 \pi \gamma^{2} B_{j}\right)^{-\frac{1}{2}} \int_{\mathbb{R}} \tilde{H}_{m_{j}}^{j}(v) \exp \left\{-\frac{\left(v-\left(\beta\left\langle\alpha_{j}, y\right\rangle+\gamma A_{j}\right)\right)^{2}}{2 \gamma^{2} B_{j}}\right\} d v \\
& =\frac{\beta^{m_{j}} \sqrt{2 \pi \gamma^{2} B_{j}}}{\sqrt{2 \pi \gamma^{2} B_{j}}} \tilde{H}_{m_{j}}^{j}\left(\left\langle\alpha_{j}, y\right\rangle+\frac{\gamma+\beta-1}{\beta} A_{j}\right) \\
& =\beta^{m_{j}} \tilde{H}_{m_{j}}^{j}\left(\left\langle\alpha_{j}, y\right\rangle+\frac{\gamma+\beta-1}{\beta} A_{j}\right) \\
& =i^{m_{j}} \tilde{H}_{m_{j}}^{j}\left(\left\langle\alpha_{j}, y\right\rangle+\frac{\sqrt{2}+i-1}{i} A_{j}\right) \\
& =i^{m_{j}} \tilde{H}_{m_{j}}^{j}\left(\left\langle\alpha_{j}, y\right\rangle-i(\sqrt{2}+i-1) A_{j}\right) .
\end{aligned}
$$

Next, applying Lemma 5.5 $\mathrm{N}$ times, we obtain a formula for generalized Fourier-Wiener function space transform of the generalized Fourier-Hermite functionals $\Phi_{\left(m_{1}, \ldots, m_{N}\right)}$.

Theorem 5.6. Let $N$ be a positive integer, for each $j=1, \ldots, N$, let $m_{j}$ be a nonnegative integer, and let

$$
\Phi_{\left(m_{1}, \ldots, m_{N}\right)}(x) \equiv \prod_{j=1}^{N} \varphi_{\left(m_{j}, j\right)}(x)=\prod_{j=1}^{N} \tilde{H}_{m_{j}}^{j}\left(\left\langle\alpha_{j}, x\right\rangle\right) .
$$

Then for each $y \in K_{a, b}[0, T]$, the generalized Fourier-Wiener function space transform $\mathcal{F}_{\sqrt{2}, i}\left(\Phi_{\left(m_{1}, \ldots, m_{N}\right)}\right)(y)$ exists and is given by the formula

$$
\begin{aligned}
\mathcal{F}_{\sqrt{2}, i}\left(\Phi_{\left(m_{1}, \ldots, m_{N}\right)}\right)(y) & =\mathcal{F}_{\sqrt{2}, i}\left(\prod_{j=1}^{N} \tilde{H}_{m_{j}}^{j}\right)\left(\left\langle\alpha_{j}, y\right\rangle\right) \\
& =i^{m_{1}+\cdots+m_{N}} \prod_{j=1}^{N} \tilde{H}_{m_{j}}^{j}\left(\left\langle\alpha_{j}, y\right\rangle-i(\sqrt{2}+i-1) A_{j}\right) \\
& =\prod_{j=1}^{N} \mathcal{F}_{\sqrt{2}, i}\left(\tilde{H}_{m_{j}}^{j}\right)\left(\left\langle\alpha_{j}, y\right\rangle\right) .
\end{aligned}
$$

In the special cases $a(t) \equiv 0$ and $b(t)=t$ on $[0, T]$, the function space $C_{a, b}[0, T]$ reduces to the Wiener space $C_{0}[0, T]$ because $A_{j}=0$ and $B_{j}=1$ for all $j=1,2, \ldots$. Hence many of the results in [1-4] are corollaries of the results in this paper. In particular:

Corollary 5.7. Let $\Phi_{\left(m_{1}, \ldots, m_{N}\right)}$ be given by (5.5). Then

$$
\mathcal{F}_{\sqrt{2}, i}\left(\Phi_{\left(m_{1}, \ldots, m_{N}\right)}\right)(y)=i^{m_{1}+\cdots+m_{N}} \Phi_{\left(m_{1}, \ldots, m_{N}\right)}(y), \quad y \in K_{0}[0, T]
$$


and

$$
\left\|\mathcal{F}_{\sqrt{2}, i}\left(\Phi_{\left(m_{1}, \ldots, m_{N}\right)}\right)\right\|_{2}=1 .
$$

Definition 5.8. Let $F \in L_{2}\left(C_{a, b}[0, T]\right)$ be given and let (4.11) be its FourierHermite series expansion with $F_{N}$ defined by (4.9) satisfying condition (4.10). Then we define the generalized Fourier-Wiener function space transform $\mathcal{F}_{\sqrt{2}, i}(F)$ of $F$ to be

$$
\mathcal{F}_{\sqrt{2}, i}(F)(x)=\lim _{N \rightarrow \infty} \mathcal{F}_{\sqrt{2}, i}\left(F_{N}\right)(x), \quad x \in C_{a, b}[0, T]
$$

if it exists; that is to say if

$$
\lim _{N \rightarrow \infty} \int_{C_{a, b}[0, T]}\left|\mathcal{F}_{\sqrt{2}, i}(F)(x)-\mathcal{F}_{\sqrt{2}, i}\left(F_{N}\right)(x)\right|^{2} d \mu(x)=0 .
$$

The following theorem is one of our main results.

Theorem 5.9. Let $F \in L_{2}\left(C_{a, b}[0, T]\right)$ be given by equation (4.11) with the generalized Fourier-Hermite coefficients of $F$ given by equation (4.5). Then the generalized Fourier-Wiener function space transform $\mathcal{F}_{\sqrt{2}, i}(F)$ of $F$ exists and is an element of $L_{2}\left(C_{a, b}[0, T]\right)$ if and only if

$$
\begin{aligned}
\lim _{N \rightarrow \infty} & \sum_{m_{1}, \ldots, m_{N}=0}^{N}\left|A_{\left(m_{1}, \ldots, m_{N}\right)}^{F}\right|^{2} \\
& \cdot \prod_{j=1}^{N} \int_{C_{a, b}[0, T]}\left|\tilde{H}_{m_{j}}^{j}\left(\left\langle\alpha_{j}, x\right\rangle-i(\sqrt{2}+i-1) A_{j}\right)\right|^{2} d \mu(x)<\infty .
\end{aligned}
$$

Furthermore if (5.9) holds, then the generalized Fourier-Hermite series expansion of $\mathcal{F}_{\sqrt{2}, i}(F)$ is given by

$$
\mathcal{F}_{\sqrt{2}, i}(F)(x)=\lim _{N \rightarrow \infty} \mathcal{F}_{\sqrt{2}, i}\left(F_{N}\right)(x)
$$

with

$$
\begin{aligned}
\mathcal{F}_{\sqrt{2}, i}\left(F_{N}\right)(x)= & \sum_{m_{1}, \ldots, m_{N}=0}^{N} A_{\left(m_{1}, \ldots, m_{N}\right)}^{F} i^{m_{1}+\cdots+m_{N}} \\
& \cdot \prod_{j=1}^{N} \tilde{H}_{m_{j}}^{j}\left(\left\langle\alpha_{j}, x\right\rangle-i(\sqrt{2}+i-1) A_{j}\right) \\
= & \sum_{m_{1}, \ldots, m_{N}=0}^{N} A_{\left(m_{1}, \ldots, m_{N}\right)}^{F} \prod_{j=1}^{N} \mathcal{F}_{\sqrt{2}, i}\left(\tilde{H}_{m_{j}}^{j}\right)\left(\left\langle\alpha_{j}, x\right\rangle\right)
\end{aligned}
$$

for each $x \in C_{a, b}[0, T]$ and each $N=1,2, \ldots$. 
Proof. First assume that $\mathcal{F}_{\sqrt{2}, i}(F)$ exists and is an element of $L_{2}\left(C_{a, b}[0, T]\right)$. For any $\epsilon>0$ we have that

$$
\int_{C_{a, b}[0, T]}\left|\mathcal{F}_{\sqrt{2}, i}(F)(x)-\mathcal{F}_{\sqrt{2}, i}\left(F_{N}\right)(x)\right|^{2} d \mu(x)<\epsilon
$$

for all sufficiently large $N$. Hence for $N$ sufficiently large,

$$
\begin{aligned}
& \left(\sum_{m_{1}, \ldots, m_{N}=0}^{N}\left|A_{\left(m_{1}, \ldots, m_{N}\right)}^{F}\right|^{2} \prod_{j=1}^{N} \int_{C_{a, b}[0, T]}\left|\tilde{H}_{m_{j}}^{j}\left(\left\langle\alpha_{j}, x\right\rangle-i(\sqrt{2}+i-1) A_{j}\right)\right|^{2} d \mu(x)\right)^{\frac{1}{2}} \\
= & \left\|\mathcal{F}_{\sqrt{2}, i}\left(F_{N}\right)\right\|_{2} \\
\leq & \left\|\mathcal{F}_{\sqrt{2}, i}(F)\right\|_{2}+\left\|\mathcal{F}_{\sqrt{2}, i}(F)-\mathcal{F}_{\sqrt{2}, i}\left(F_{N}\right)\right\|_{2} \\
\leq & \left\|\mathcal{F}_{\sqrt{2}, i}(F)\right\|_{2}+\epsilon
\end{aligned}
$$

and thus by condition (5.9) holds.

To proof the converse, suppose that condition (5.9) holds. For integers $M$ and $N$ with $M>N \geq 1$, let

$$
I_{M}=\left\{\left(m_{1}, \ldots, m_{M}\right): m_{1}, \ldots, m_{M}=0,1, \ldots, M\right\}
$$

and let

$I_{N}=\left\{\left(m_{1}, \ldots, m_{M}\right): m_{1}, \ldots, m_{N}=0,1, \ldots, N\right.$ and $\left.m_{N+1}=\cdots=m_{M}=0\right\}$.

Then it follows that

$$
\begin{aligned}
& \int_{C_{a, b}[0, T]}\left|\mathcal{F}_{\sqrt{2}, i}\left(F_{M}\right)(x)-\mathcal{F}_{\sqrt{2}, i}\left(F_{N}\right)(x)\right|^{2} d \mu(x) \\
&= \int_{C_{a, b}[0, T]} \mid \sum_{I_{M}-I_{N}} A_{\left(m_{1}, \ldots, m_{M}\right)}^{F} i^{m_{1}+\cdots+m_{M}} \\
&\left.\cdot \prod_{j=1}^{M} \tilde{H}_{m_{j}}^{j}\left(\left\langle\alpha_{j}, x\right\rangle-i(\sqrt{2}+i-1) A_{j}\right)\right|^{2} d \mu(x) \sum_{I_{M}-I_{N}}\left|A_{\left(m_{1}, \ldots, m_{M}\right)}^{F} i^{m_{1}+\cdots+m_{M}}\right|^{2} \\
&=\left.\sum_{m_{1}, \ldots, m_{M}=0}^{M}\left|A_{\left(m_{1}, \ldots, m_{M}\right)}^{F} \int_{C_{a, b}[0, T]}\right| \tilde{H}_{m_{j}+\cdots+m_{M}}^{j}\left(\left\langle\alpha_{j}, x\right\rangle-i(\sqrt{2}+i-1) A_{j}\right)\right|^{2} d \mu(x) \\
& \cdot \prod_{j=1}^{M} \int_{C_{a, b}[0, T]}\left|\tilde{H}_{m_{j}}^{j}\left(\left\langle\alpha_{j}, x\right\rangle-i(\sqrt{2}+i-1) A_{j}\right)\right|^{2} d \mu(x)
\end{aligned}
$$




$$
\begin{aligned}
-\sum_{m_{1}, \ldots, m_{N}=0}^{N} \mid & \left.A_{\left(m_{1}, \ldots, m_{N}\right)}^{F} i^{m_{1}+\cdots+m_{N}}\right|^{2} \\
& \cdot \prod_{j=1}^{N} \int_{C_{a, b}[0, T]}\left|\tilde{H}_{m_{j}}^{j}\left(\left\langle\alpha_{j}, x\right\rangle-i(\sqrt{2}+i-1) A_{j}\right)\right|^{2} d \mu(x)
\end{aligned}
$$

which goes to 0 as $M, N \rightarrow \infty$. Hence $\left\{\mathcal{F}_{\sqrt{2}, i}\left(F_{N}\right)\right\}_{N=1}^{\infty}$ is a Cauchy sequence in $L_{2}\left(C_{a, b}[0, T]\right)$ and since $L_{2}\left(C_{a, b}[0, T]\right)$ is complete,

$$
\mathcal{F}_{\sqrt{2}, i}(F)(x)=\lim _{N \rightarrow \infty} \mathcal{F}_{\sqrt{2}, i}\left(F_{N}\right)(x), \quad x \in C_{a, b}[0, T]
$$

exists and is an element of $L_{2}\left(C_{a, b}[0, T]\right)$.

Remark 5.10. The main result in [10], namely Theorem 6 on page 1385, as well as the main results of [3], namely Theorem 1 on page 103 , follows immediately from Theorem 5.9 above by choosing $a(t) \equiv 0$ and $b(t)=t$ on $[0, T]$, and $\beta=i$ and $\gamma=\sqrt{2}$.

Corollary 5.11. Suppose that condition (5.9) holds. If $a(t) \equiv 0$ on $[0, T]$, then

$$
\left\|\mathcal{F}_{\sqrt{2}, i}(F)\right\|_{2}^{2}=\lim _{N \rightarrow \infty} \sum_{m_{1}, \ldots, m_{N}=0}^{N}\left|A_{\left(m_{1}, \ldots, m_{N}\right)}^{F}\right|^{2}=\|F\|_{2}^{2} .
$$

\section{References}

[1] R. H. Cameron, Some examples of Fourier-Wiener transforms of analytic functionals, Duke Math. J. 12 (1945), 485-488.

[2] R. H. Cameron and W. T. Martin, Fourier-Wiener transforms of analytic functionals, Duke Math. J. 12 (1945), 489-507.

[3] _ Fourier-Wiener transforms of functionals belonging to $L_{2}$ over the space $C$, Duke Math. J. 14 (1947), 99-107.

[4] _ The orthogonal development of non-linear functionals in series of FourierHermite functionals, Ann. of Math. 48 (1947), 385-392.

[5] R. H. Cameron and D. A. Storvick, An L 2 analytic Fourier-Feynman transform, Michigan Math. J. 23 (1976), no. 1, 1-30.

[6] K. S. Chang, B. S. Kim, and I. Yoo, Integral transform and convolution of analytic functionals on abstract Wiener space, Numer. Funct. Anal. Optim. 21 (2000), no. 1-2, 97-105.

[7] S. J. Chang and D. M. Chung, Conditional function space integrals with applications, Rocky Mountain J. Math. 26 (1996), no. 1, 37-62.

[8] S. J. Chang, J. G. Choi, and D. Skoug, Integration by parts formulas involving generalized Fourier-Feynman transforms on function space, Trans. Amer. Math. Soc. 355 (2003), no. 7, 2925-2948.

[9] S. J. Chang and D. L. Skoug, Generalized Fourier-Feynman transforms and a first variation on function space, Integral Transforms Spec. Funct. 14 (2003), no. 5, 375393.

[10] B. S. Kim and D. Skoug, Integral transforms of functionals in $L_{2}\left(C_{0}[0, T]\right)$, Rocky Mountain J. Math. 33 (2003), no. 4, 1379-1393. 
[11] Y. J. Lee, Integral transforms of analytic functions on abstract Wiener spaces, J. Funct. Anal. 47 (1982), no. 2, 153-164.

[12] - Unitary operators on the space of $L^{2}$-functions over abstract Wiener spaces, Soochow J. Math. 13 (1987), no. 2, 165-174.

[13] D. Skoug and D. Storvick, A survey of results involving transforms and convolutions in function space, Rocky Mountain J. Math. 34 (2004), no. 3, 1147-1175.

[14] E. Nelson, Dynamical Theories of Brownian Motion (2nd edition), Math. Notes, Princeton University Press, Princeton, 1967.

[15] J. Yeh, Stochastic Processes and the Wiener Integral, Marcel Dekker, Inc., New York, 1973.

[16] _ Singularity of Gaussian measures on function spaces induced by Brownian motion processes with non-stationary increments, Illinois J. Math. 15 (1971), 37-46.

Seung Jun Chang

Department of Mathematics

DANKOOK UNIVERSITY

Cheonan 330-714, Korea

E-mail address: sejchang@dankook.ac.kr

Hyun Soo Chung

Department of Mathematics

DANKOOK UNIVERSITY

Cheonan 330-714, Korea

E-mail address: hschung@dankook.ac.kr 